\title{
Performance Analysis of Rayleigh and Rician Fading Channel Model
}

\author{
Jaspreet kaur $^{1}$, Megha Khanna ${ }^{2}$ and Gurjot Singh Gaba ${ }^{3 *}$ \\ ${ }^{1,2,3}$ Discipline of Electronics and Communication Engineering \\ Lovely Professional University, Phagwara, Punjab, India - 144411 \\ 1jaspreetkaursandhu589@gmail.com, ${ }^{2}$ kmegha014@gmail.com, \\ Corresponding Author - ${ }^{3}$ er.gurjotgaba@gmail.com
}

\begin{abstract}
This paper presents an overview of performance analysis for Rayleigh and Rician fading channel models. Analysis of the channel models are virtually carried out in terms of outage probability, source probability, Bit Error Rate and Signal to Noise Ratio. Source and outage probability has been computed to compare the signal detection characteristics under different models. Further, channel estimation has been done via different methods which include least square (LS), Least Square Modified (LSMod), Minimum Mean Square Error (MMSE). The results obtained can be used to optimize the performance of the network.
\end{abstract}

Keywords: Rayleigh fading, Rician fading, Bit Error Rate, LS, LSMod, MMSE

\section{Introduction}

When a signal travels from transmitter to the receiver, the characteristics of signal changes when it travels due to reflection and refraction, hence suffer from multipath fading. There are several factors which influence such as how the channel behaves such as climatic conditions changes, speed of transmission and reception, distance between them etc. The instantaneous signal strength can be calculated by large and small scale models where large scale models help in depiction of average received signal strength which depends on the distance between transmitter and receiver. The small scale model on the other hand represents the variations in the signal strength. However, error measurement of the channel depends upon Line of Sight, reflections, refraction, diffraction and scattering caused by an object before it arrives at receiver. Fading occurs in wireless communication due to different components which arrive at receiver at different time which may lead to phase difference between transmitted and received components and may be frequency shifted also. Channel estimation is required in MIMO-OFDM which includes estimation of bits transmitted as well as received. Estimation of Rayleigh and Rician channel models has been performed in this paper. Assumption in the Rayleigh fading channel is that fading occurs due to variations in amplitudes and phases. The simulation has been carried out in MATLAB and analysis is performed in terms of outage, source probability, BER and SNR.

\section{Types of Channel Model}

(i) Rayleigh Fading Channel:

Rayleigh distribution model is a model where there is no line of sight communication between transmitter and receiver. It is a special case of stochastic model [1]. When

* Corresponding Author 
scattering of dominant component does not take place, then there will be zero mean and phase between 0 and $2 \pi$ radian.

(ii) Rician Fading Channel:

The small scale fading distribution is said to be Rician when there is line of sight propagation. All the multipath components are superimposed on a dominant signal in this fading. It usually occurs when one of path is comparatively stronger than the other. As the dominating component goes into more fading, the characteristics of the signal changes from Rician to Rayleigh distribution.

\section{Algorithms for Channel Estimation}

(i) Least Square:

This approach finds the approximate solution in minimizing the sum of squares of the errors which were present in unit equation.

$$
H(l s)=A B
$$

Where, $\quad A=$ input

$\mathrm{B}=$ output

$\mathrm{H}(\mathrm{ls})=$ channel matrix;

(ii) Minimum Mean Square:

It is a common method to estimate quality of channel. It is generally preferred for signals with low SNR values. This model is estimated by using the equation

$$
M S E=E\left\{\left(H-H(l s)^{H(H-H(l s)}\right\}\right\}
$$

Where,

$\mathrm{H}=$ channel matrix

$\mathrm{H}(\mathrm{ls})=\mathrm{LS}$ algorithm's channel matrix

MSE $=$ Mean Square Error

(iii) Least Square Modified:

This is the best approach which is preferred most of the times like in data fitting. It minimizes the sum of squared residuals. The equation represents the value:

$$
\text { Hest }=\frac{\left(\text { Hest }_{(i)}+\text { Hest }_{(i+1)}\right)}{2}
$$

Where

Hest $(i)=$ estimated channel matrix for $\mathrm{i}^{\text {th }}$ value

Hest $_{(i+1)}=$ estimated channel matrix for $(i+1)^{\text {th }}$ value

Hest $=$ estimated channel matrix

\section{Performance Analysis: Rayleigh Fading Channel Model}

The received signal can be expressed:

$$
s(t)=a_{i} \sum_{i=1}^{n} \cos \left(\omega_{c} t+\omega_{d i} t+\emptyset_{i}\right)
$$

Where,

$\mathrm{n}$ is the number of paths, $\emptyset=[0,2 \pi]$

This equation is rewritten in terms of phase and quadrature component:

$$
s(t)=I(t) \cos \omega_{c} t+\emptyset_{i}(t) \sin \omega_{c} t
$$

Where,

$$
I(t)=\sum_{i=1}^{n} a_{i} \cos \left(\omega_{d i}(t)+\emptyset_{i}\right)
$$


When $\mathrm{n}$ is large, $\mathrm{f}(\mathrm{r})$ can be written as

$$
\emptyset(t)=\sum_{i=1}^{n} a_{i} \sin \left(\omega_{d i}(t)+\emptyset_{i}\right)
$$

$$
f(r)=\frac{r}{\sigma^{2}} e^{-r 2 / 2 \sigma 2}
$$

Where $\sigma^{2}$ is time-averaged power of received signal before envelope detection. As it is clear from the Figure 1, that with increase in the threshold power, there is increase in outage probability i.e. decrease in probability for better detection of the signal [2].

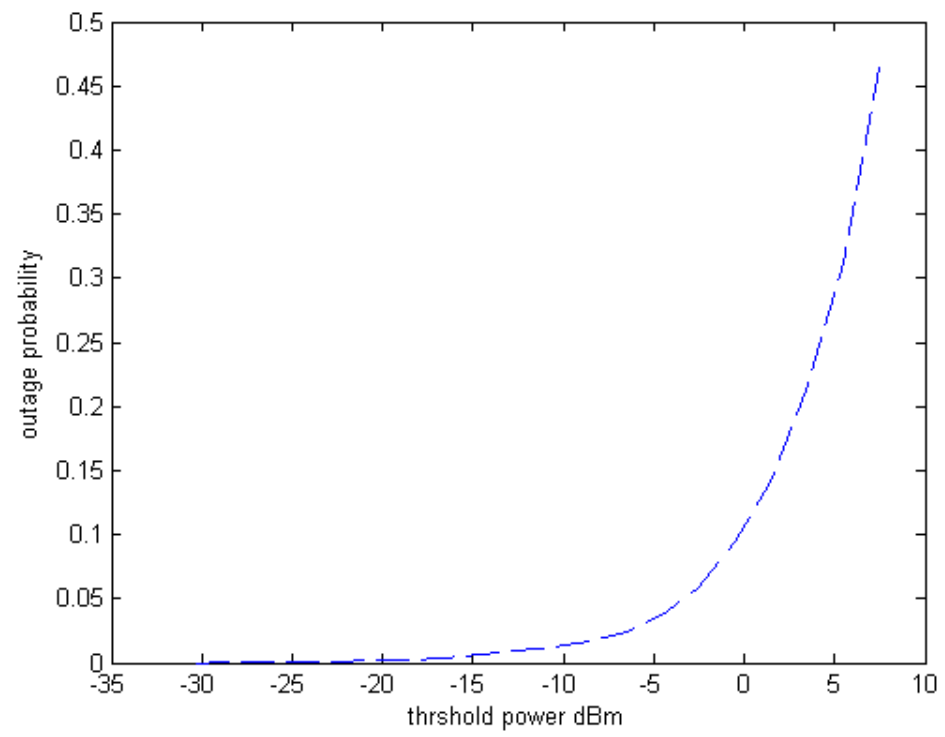

Figure 1. Outage Probability for Rayleigh Fading Channel [2]

BER is defined as the bits in error with respect to total bits transmitted through the channel. BER should be preferably low. In order to do channel estimation in OFDM, the optimized parameters need to be considered for proper synchronization. Let us consider $\mathrm{N}$ $=64$ and pilot $=64$ for simulation in MATLAB.

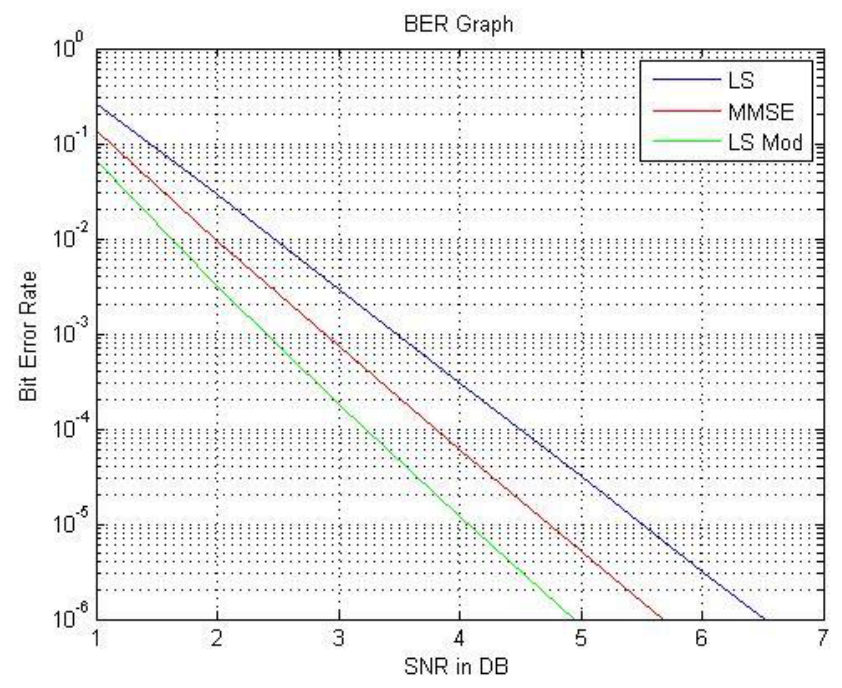

Figure 2. SNR vs. BER [3] 
Figure 2 clearly depicts the BER vs. SNR plot using the three methods namely LS, MMSE, LS Mod [3]. LS Mod has the least BER so it is better method for channel estimation in Rayleigh fading.

\section{Performance Analysis: Rician Fading Channel Model}

For Rician channel [4] the expression of signal received can be given as:

$$
\begin{array}{r}
s(t)=\sum_{i=1}^{n-1} a_{i} \cos \left(\omega_{c} t+\omega_{d i}(t)+\varphi i\right)+k_{d} \cos \left(\omega_{c} t+\omega_{d} t\right) \\
\omega_{d} \text { is doppler shift along line of sight path }
\end{array}
$$

Where, $\mathrm{k}_{\mathrm{d}}$ is strength of direct component

$$
f(r)=\frac{r}{\sigma^{2}} e^{\left.\left\{\frac{-r^{2}+k a^{2}}{2 \sigma^{2}}\right] I \frac{r^{2} a^{d}}{\sigma^{2}}\right\}}
$$

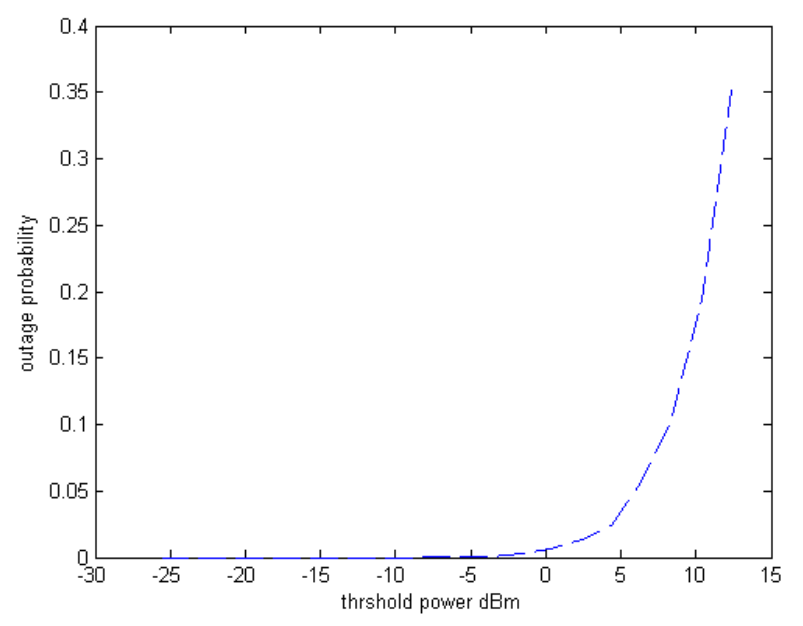

Figure 3. Outage Probability for Rician Fading Channel [2]

Let $\mathrm{N}=64$ and pilot $=64$. Now the simulation of this channel clearly shows the plot of BER vs. SNR in the Figure 4.

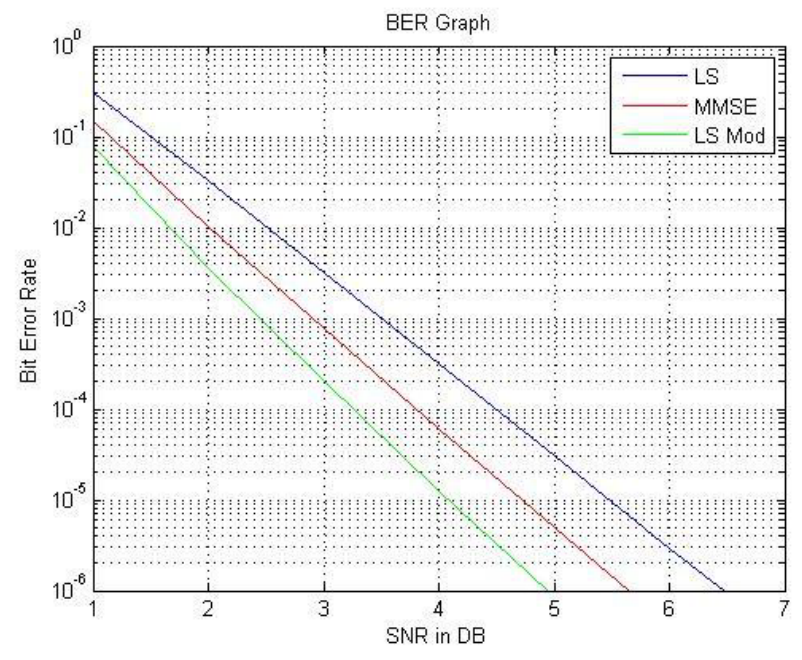

Figure 4. SNR vs. BER [3]

Table 1 portrays the comparison of BER values for different techniques considered in Rician and Rayleigh channel models. It is found that for low SNR, BER is less in 
Rayleigh model as compared to Rician model. The preferred technique is LS Mod as it encounters the minimum BER in Rayleigh modelling for channel estimation.

Table 1. Comparison of BER [3]

\begin{tabular}{|c|c|c|c|}
\hline Channel model & LS algorithm & MMSE algorithm & LS Mod \\
\hline Rayleigh & 0.2664 & 0.1385 & 0.0666 \\
\hline Rician & 0.3070 & 0.1516 & 0.0768 \\
\hline
\end{tabular}

\section{Conclusion}

In this paper, we have done channel estimation for MIMO-OFDM systems of the two different channel models namely Rayleigh and Rician. This was performed via three methods which include LS, MMSE and LS Mod. LS method is the simplest method but does not go well where low SNR values come in picture. MMSE can be used for lower SNR but is very complex. So LS Mod is preferred over the other two. Moreover the Bit Error Rate (BER) for Rayleigh is less in comparison with Rician. But on other hand, Outage probability for Rician fading channel is less than the Rayleigh due to Line of sight component present between the transmitter and receiver.

\section{References}

[1] L. Hanzo, Y. Akhtman, L. Wang and M. Jiang, "MIMO-OFDM for LTE, WIFI and WIMAX", WileyIEEE Press, (2010).

[2] S. Kumar, P. K. Gupta, G. Singh and D. S. Chauhan, "Performance analysis of Rayleigh and Rician fading channel models using MATLAB simulation”, I.J Intelligent Systems and Applications, vol. 9, (2013), pp. 94-102.

[3] K. Vidhya and R. K. S. Kumar, "BER performance of AWGN, Rayleigh and Rician Channel," International Journal of Advanced Research in Computer and Communication Engineering, vol. 2, no. 5, (2013), pp. 2058-2067.

[4] Theodore S. Rappaport, "Wireless communications principles and practice," Pearson, Second edition, (2001).

\section{Authors}

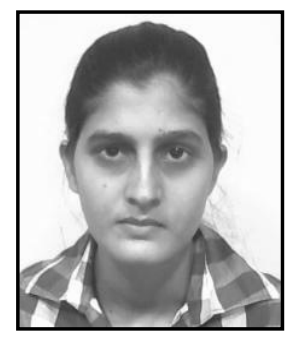

Jaspreet Kaur, is currently pursuing her Masters in Electronics and Communication Engineering from Lovely Professional University. She is working in this field since 2015 and has potential to resolve several problems of industry through her expertise.

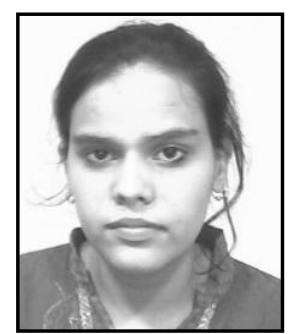

Megha, is currently pursuing her Masters in Electronics and Communication Engineering from Lovely Professional University. She is working in this field since 2015 and has potential to resolve several problems of industry through her expertise. 


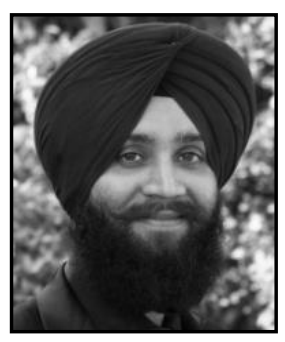

Gurjot Singh Gaba, is currently pursuing Ph.D. in Electronics \& Electrical Engineering with Spl. in Cryptography and Network Security of WSN and IoT's. He is working as an Asst. Prof. in Lovely Professional University, India since 2011. His research interest includes Wireless Sensor Networks, Computer Networks, Optical Communications and Cryptography. He is a reviewer of SCIE and Scopus Indexed Journals. He has recently been appointed as Editor of IJEEE journal. He is a member of many technical bodies including ISCA, IAENG, IACSIT, CSI, and ISTE. He is an author of six International books and more than two dozen research papers. 